\title{
Synthesis, Characterization and Application of a Molecularly Imprinted Polymer in Selective Adsorption of Abacavir from Polluted Water
}

\author{
Sharlott N. Qwane, Phumlane S. Mdluli and Lawrence M. Madikizela* \\ Department of Chemistry, Durban University of Technology, P.O. Box 1334, Durban, 4000, South Africa.
}

Received 15 November 2019, revised 17 March 2020, accepted 30 April 2020

\begin{abstract}
Abacavir is an antiretroviral drug (ARVD), which has been identified as a water pollutant. To find a selective adsorbent for its extraction from water, a molecularly imprinted polymer (MIP) is proposed for its selective uptake. A 24-hour bulk polymerization process was performed for imprinting abacavir with aliquat 336 and ethylene glycol dimethacrylate, as functional monomer and cross-linking agent, respectively. Uptake of abacavir from $10 \mathrm{~mL}$ of aqueous solutions was performed with $40 \mathrm{mg}$ of MIP at pH 5 within $60 \mathrm{~min}$ of contact time. MIP selectively adsorbed abacavir from water in the presence of other ARVDs (tenofovir disoproxil and efavirenz). The maximum adsorption capacity obtained for abacavir was $5.98 \mathrm{mg} \mathrm{g}^{-1}$. The adsorption mechanism was best described by the Freundlich isotherm and pseudo-second-order kinetic model, which were translated to multilayer coverage and chemisorption influenced by electrostatic attractions, respectively. The extraction efficiencies achieved for abacavir in wastewater influent, effluent and estuarine water were 67,74 and $76 \%$, respectively. The synthesized MIP can be reused at least 10 consecutive times for adsorption of abacavir from polluted water without losing its extraction efficiency. This is the first study to report the application of aliquat 336 as the functional monomer in the synthesis of MIP for selective extraction of abacavir from water.
\end{abstract}

KEYWORDS

Abacavir, adsorption, aqueous solutions, extraction efficiency.

\section{Introduction}

Abacavir belongs to the class of non-nucleoside reverse transcriptase inhibitors used by humans for the treatment of retroviral infections, primarily human immunodeficiency virus type $1 .{ }^{1}$ About $18 \%$ of abacavir has been reported to be excreted from humans as part of faecal matter $(16 \%)$ and urine $(2 \%){ }^{2}$ This is the main route for the transfer of abacavir from sewage into the wastewater treatment plants (WWTPs). Due to the polar groups present in abacavir and its water solubility (Table 1), the drug is expected to be incompletely removed from water during the purification process. Mostly, the effluent from the WWTPs is discharged into the rivers and in some cases directly into the sea. Thus far, few scientific articles that report the occurrence of abacavir in WWTPs and surface water have been published. ${ }^{3-8}$ In South Africa, traces of abacavir have been detected, with other antiretroviral drugs (ARVDs), in the surface water but the concentrations were below the quantitation limits. ${ }^{7}$ Higher quantities of up to $14 \mu \mathrm{g} \mathrm{L}^{-1}$ were found in the WWTP influent, while the levels of abacavir in the effluents were below the limit of quantitation. ${ }^{8}$

Affordable analytical methods that are sensitive and selective for abacavir routine analysis in water are not readily available. The quantification of abacavir in water requires high performance liquid chromatography (HPLC) analysis after a suitable sample preparation step, such as solid-phase extraction (SPE). SPE is a sample preparation technique that is well exploited for the extraction and pre-concentration of pharmaceuticals in water. ${ }^{9}$ Several adsorbents, which include activated carbons, clays, chitosan and molecularly imprinted polymers (MIPs), are

*To whom correspondence should be addressed. E-mail: lawrencem2@ dut.ac.za well described in literature for the removal of pharmaceuticals in water. ${ }^{10,11}$ However, the SPE sorbents that are available commercially tend to be selective to a wide range of compounds, which could be problematic when the analysis of a single analyte is required.

Among these adsorbents, MIPs are known for their ease of preparation, excellent selectivity, outstanding adsorption capabilities and re-usability. ${ }^{11}$ Therefore, MIPs which are synthetic polymers with highly specific recognition ability for target molecules have been evaluated for the adsorption of organics in water and applied as selective sorbents in SPE for various pharmaceuticals. ${ }^{12-14}$ The synthetic approach of MIPs involves the formation of a complex, between the functional monomer and template (usually a target molecule), through covalent or non-covalent interactions in the presence of a cross-linking agent. ${ }^{15}$ Thereafter, the template is removed through the exhaustive washing of the synthesized polymer. This creates the binding sites that are complementary to the template's size, shape, and position of the functional groups, and consequently allows for its selective uptake. ${ }^{15}$ Therefore, the functional monomer plays a crucial role in molecular imprinting. Two functional monomers reported in literature for the imprinting of abacavir are acrylic acid ${ }^{16}$ and itaconic acid. ${ }^{17}$

In this study, a MIP for abacavir was synthesized in the presence of ethylene glycol dimethacrylate (EGDMA) as crosslinking agent using aliquat 336 and toluene as the functional monomer and porogenic solvent, respectively. Thereafter, the synthesized MIP was evaluated for the selective removal of abacavir from polluted water. The application of aliquat 336 as the functional monomer during molecular imprinting was motivated by a need to perform procedures that adhere to green 
Table 1 Chemical structures and physicochemical properties of abacavir, tenofovir disoproxil, efavirenz and aliquat 336 used in selective adsorption.

\begin{tabular}{|c|c|c|c|c|}
\hline Compound & Chemical structure & $\begin{array}{l}\text { Molecular weight } \\
\qquad / \mathrm{g} \mathrm{mol}^{-1}\end{array}$ & $\mathrm{pKa}$ & $\begin{array}{l}\text { Water solubility } \\
\qquad / \mathrm{mg} \mathrm{mL}^{-1}\end{array}$ \\
\hline Abacavir & & 286 & 5.06 & 77 \\
\hline Tenofovir disoproxil & & 519 & 4.13 & 13.14 \\
\hline Efavirenz & & 316 & -1.5 & 0.00855 \\
\hline Aliquat 336 & & 404 & - & Insoluble \\
\hline
\end{tabular}

Physicochemical properties obtained from the literature. ${ }^{1,21}$ - Information not available.

chemistry principles. Aliquat 336 was considered as a suitable replacement of the traditional functional monomers as it is an ionic liquid with excellent extracting capabilities for organic ${ }^{18}$ and inorganic materials. ${ }^{19}$ In general, ionic liquids are known as green solvents, with advantages such as excellent dissolution ability, high thermal stability and easy recycling. ${ }^{20}$ The synthesized MIP was able to adsorb abacavir selectively in wastewater and estuarine water, also polluted with other ARVDs such as tenofovir disoproxil and efavirenz (general properties are given in Table 1), through electrostatic attraction.

\section{Experimental}

\subsection{Chemicals and Reagents}

Abacavir (98\%), aliquat 336 ( $\geq$ E98 \%), 1,1-azobis-(cyclohexanecarbonitrile) (98\%), EGDMA (98\%), orthophosphoric acid (85\%), HPLC grade solvents, which included methanol $(\geq 99.9 \%)$, acetonitrile ( $\geq 99.9 \%)$ and toluene $(99.7 \%)$ ), were all purchased from Sigma-Aldrich (Johannesburg, South Africa). Glacial acetic acid (100\%) was purchased from Merk Chemicals (Pty) Ltd (Johannesburg, South Africa). Tenofovir disoproxil and efavirenz were supplied by LEAP Chem (Hangzhou, China) and J \& H Chem Co. Ltd (Hangzhou, China), and purchased through DLD Scientific (Durban, South Africa), respectively.

\subsection{Chromatographic Separation}

Chromatographic separation was performed on a HPLC system from Shimadzu Corporation (Kyoto, Japan). The HPLC system consisted of an online mobile phase degasser unit (Model: DGU-20A3), $20 \mu \mathrm{L}$ sample loop, pump (Model: LC-20AB) and UV/vis detector (Model: SPD-20A) monitoring abacavir at $215 \mathrm{~nm}$. The mobile phase used for chromatographic analysis performed on SGE $\mathrm{C}_{18}$ column $(250 \mathrm{~mm} \times 4.6 \mathrm{~mm} \times$ $5 \mu \mathrm{m})$ from Trajan (Milton Keynes, United Kingdom) was a mixture of methanol and $0.05 \%$ orthophosphoric acid in water $(\mathrm{pH} 3)$ at a ratio of 60:40 v/v flowing at a rate of $1 \mathrm{~mL} \mathrm{~min}^{-1}$. The instrument was equipped with Shimadzu LC solutions software for data collection and processing.

\subsection{Synthesis of Polymers}

Synthesis of MIP was performed using a method that was initially reported by Duan and coworkers ${ }^{22}$ and modified in our research group for various pharmaceuticals. ${ }^{12}$ In this case, two polymerization steps were performed at $65^{\circ} \mathrm{C}$. In the first step, EGDMA homopolymer was synthesized by mixing $5.03 \mathrm{mmol}$ of EGDMA with $0.085 \mathrm{mmol}$ of 1,1-azobis-(cyclohexanecarbonitrile) (an initiator) in a $250 \mathrm{~mL}$ round-bottom flask containing a magnetic stirrer followed by the addition of $50 \mathrm{~mL}$ toluene. The mixture was purged with nitrogen for $15 \mathrm{~min}$ to remove oxygen 
and dissolved gasses, followed by sealing the flask under inert atmosphere. Polymerization on an oil bath with constant stirring at a rate of $300 \mathrm{rpm}$ was allowed to occur at $65^{\circ} \mathrm{C}$ for $8 \mathrm{~h}$. This resulted in the formation of monodispersed EGDMA homopolymer used as a main reactant in the following reaction step. In the second step, $0.75 \mathrm{mmol}$ of abacavir, $1.83 \mathrm{mmol}$ of aliquat 336, $4.52 \mathrm{mmol}$ of EGDMA and $0.085 \mathrm{mmol}$ of 1,1-azobis-(cyclohexanecarbonitrile) were dissolved in $50 \mathrm{~mL}$ of acetonitrile/ toluene $(50: 50 \mathrm{v} / \mathrm{v})$. The resulting mixture was transferred into the first reaction flask and deoxygenated by purging with nitrogen for $15 \mathrm{~min}$. Polymerization, which resulted in the formation of the bulk polymer, was performed in the oil bath at $65{ }^{\circ} \mathrm{C}$ for $22 \mathrm{~h}$. The elution of the template from the resulting polymer was performed by washing the polymer repeatedly with a mixture of methanol:acetic acid $(9: 1 \mathrm{v} / \mathrm{v})$ until abacavir was not detected by the HPLC system. Thereafter, the polymer was rinsed three times with high purity methanol. A similar procedure was followed for the synthesis and treatment of a non-imprinted polymer (NIP) in the absence of the template molecule, namely abacavir. Both polymers were milled on a planetary ball mill (Retsch PM 100) from Retsch GmbH (Haan, Germany) followed by sieving using a mechanical sieve shaker (Retsch AS 200) from Retsch GmbH (Haan, Germany) for collection of particles that ranged from 25 to $50 \mu \mathrm{m}$ in diameter.

\subsection{Characterization of Polymers}

The thermal stability of both MIP and NIP was investigated using thermogravimetric analysis (TGA)/differential scanning calorimetry (DSC) 1 Star ${ }^{\mathrm{e}}$ system obtained from Mettler Toledo (Columbus, USA). The polymers were heated from $25^{\circ} \mathrm{C}$ to $1000{ }^{\circ} \mathrm{C}$ under nitrogen atmosphere flowing at a rate of $100 \mathrm{~mL} \mathrm{~min}{ }^{-1}$. The heating rate was maintained at $10^{\circ} \mathrm{C} \mathrm{min}^{-1}$.

Further characterization was performed on Agilent VNMRS Wide Bore $500 \mathrm{MHz}$ nuclear magnetic resonance (NMR) spectrometer with a $1 \mathrm{H}$ frequency of $500 \mathrm{MHz}$ and a $13 \mathrm{C}$ frequency of $125 \mathrm{MHz}$. A 4 mm HX Magic-angle-spinning (MAS) probe was used to collect the NMR spectra. A MAS rate of 10000 revolutions per second $(10 \mathrm{kHz})$ was used for both polymers. The crosspolarization $(\mathrm{CP})$ spectra were recorded at $25{ }^{\circ} \mathrm{C}$ with proton decoupling using a recycle delay of $10 \mathrm{~s}$. The $\mathrm{CP}$ pulse power parameters were optimized for the Hartmann-Hahn match using a glycine standard sample. The radio frequency fields for the match were $\gamma \mathrm{CB} 1 \mathrm{C}=\gamma \mathrm{HB} 1 \mathrm{H} \approx 55 \mathrm{kHz}$. The contact time for cross-polarization was optimized to $2.0 \mathrm{~ms}$. Finally, the spectra for polymers were Fourier transformed, baseline corrected, integrated and the relevant peaks marked.

Surface area, pore size and pore volume of both polymers were characterized using a Brunauer-Emmett-Teller (BET) Micromeritics TriStar 3000 system from Micromeritics Instrument Corp. (Norcross, USA).

\subsection{Optimization of Adsorption Experiments}

Several factors that could affect the adsorption of abacavir from polluted aqueous solutions were optimized in batch mode. Such parameters were sample $\mathrm{pH}$, polymer amount, concentration of target compound and contact time. Only one parameter was optimized at a time, while keeping other adsorption parameters constant. All experiments were conducted in triplicate $(\mathrm{n}=$ 3 ) and the average results were computed. Extraction efficiency and adsorption capacity used to measure the extent of adsorption were calculated using Equations (1) and (2), respectively.

$$
\text { Extraction efficiency }(\%)=\frac{\left(C_{\mathrm{o}}-\mathrm{C}_{\mathrm{e}}\right)}{\mathrm{C}_{\mathrm{o}}} \times 100
$$

$$
\text { Adsorption capacity }\left(\mathrm{mg} \mathrm{g}^{-1}\right)=\frac{\left(\mathrm{C}_{\mathrm{o}}-\mathrm{C}_{\mathrm{e}}\right) \mathrm{V}}{\mathrm{W}} \times 100
$$

where $\mathrm{Co}$ is the initial concentration $\left(\mathrm{mg} \mathrm{L}^{-1}\right)$ of abacavir before the adsorption process, Ce is the final concentration $\left(\mathrm{mg} \mathrm{L}^{-1}\right)$ of abacavir remaining after adsorption, $\mathrm{V}$ is the volume of solution $(\mathrm{L})$ and $\mathrm{W}$ is the mass of polymer $(\mathrm{g})$.

\subsection{Selectivity Studies}

The selectivity of MIP was tested by performing the adsorption of abacavir from water using the optimum conditions in the presence of tenofovir disoproxil and efavirenz. This was done by transferring $10 \mathrm{~mL}$ of deionized water spiked with a mixture of abacavir, tenofovir disoproxil and efavirenz $\left(50 \mu \mathrm{g} \mathrm{L}^{-1}\right.$ of each drug) into a vial containing $40 \mathrm{mg}$ of MIP. The contents of the vial were stirred ( $350 \mathrm{rpm})$ for $60 \mathrm{~min}$ at room temperature and transferred to $3 \mathrm{~mL}$ SPE tubes from Biotage (Uppsala, Sweden). Two polypropylene frits with pore size of $10 \mu \mathrm{m}$ from Biotage (Uppsala, Sweden) were fitted at the bottom of the cartridge and above the MIP to safeguard against the adsorbent loss. Liquid solution was allowed to go to waste. The adsorbed compounds were eluted from the MIP with $4 \mathrm{~mL}$ mixture of methanol:acetic acid (80:20; v:v) flowing at $0.5 \mathrm{~mL} \mathrm{~min}^{-1}$ and analyzed using HPLC.

\subsection{Regeneration of Molecularly Imprinted Polymer}

After each adsorption process, a MIP loaded with abacavir from aqueous solution was regenerated by desorbing the drug with a mixture of methanol $(80 \%)$ and acetic acid $(20 \%)$ followed by methanol (100\%). Thereafter, the same MIP was applied for further adsorption of abacavir from deionized water spiked with $50 \mu \mathrm{g} \mathrm{L}^{-1}$ of the drug. The optimized adsorption conditions were applied in each case, with each experiment conducted in triplicate. The extraction efficiency was evaluated. These adsorption and desorption experiments were performed repeatedly for ten consecutive times.

\subsection{Adsorption of Abacavir from Polluted Water}

The optimized adsorption parameters were applied in the selective removal of abacavir from wastewater and estuarine water. Wastewater samples (raw influent after screening for removal of solid particles and final effluent after chlorination for disinfection) were collected from Northern WWTP (GPS coordinates: -29.795668 ; 30.999130) located in Durban (South Africa) using glass bottles. Estuarine water was collected in Durban Blue Lagoon (Umgeni estuary) (GPS coordinates: -29.811566; 31.038487), South Africa. These study sites have been previously reported to be polluted with a wide range of pharmaceuticals including antibiotics, ${ }^{23,24}$ non-steroidal anti-inflammatory drugs $^{25,26}$ and ARVDs. $8,14,27$ The collected samples were preserved by storing in a cooler box with ice during their transportation into the laboratory. Upon arrival in the laboratory, the collected samples were filtered through a $0.45 \mu \mathrm{m}$ filter paper supplied by Membrane Solutions (Chiba-ken, Japan) and stored at $4{ }^{\circ} \mathrm{C}$. The adsorption experiments were performed within one week. Prior to adsorption experiments, abacavir was quantified in collected wastewater (influent and effluent) and estuarine samples. The detected concentrations in wastewater influent, effluent and estuarine water were 41,24 and $22 \mu \mathrm{g} \mathrm{L}^{-1}$, respectively. Thereafter, the same water samples were spiked with abacavir to achieve a final concentration of $50 \mu \mathrm{g} \mathrm{L} \mathrm{L}^{-1}$ of target compound. The adsorption process was performed on $10 \mathrm{~mL}$ spiked water solution over $60 \mathrm{~min}$ using $40 \mathrm{mg}$ of MIP. After 60 min of contact (with constant stirring at $350 \mathrm{rpm}$ ) between 
water solution and MIP, the abacavir that remained in solution was quantified on HPLC and the extraction efficiency was computed.

\section{Results and Discussion}

\subsection{Synthesis of Molecularly Imprinted Polymer}

The adsorption of organic compounds using MIPs is strongly influenced by the nature of the functional monomer used in polymerization reaction. In this study, a MIP was synthesized using aliquat 336 as the functional monomer. This functional monomer was selected based on its ability to improve the greenness of the synthetic procedure and the end-product (MIP) as ionic liquids are categorized as green chemicals. As shown in Table 1, unlike the functional monomers reported in literature, aliquat 336 does not has any functional groups in its molecular structure. However, its permanent positive charge is expected to be the driving force during the binding with abacavir. Other reagents used during the synthesis of MIP are EGDMA, toluene and 1,1-azobis-(cyclohexanecarbonitrile) which are common chemicals applied in the preparation of MIPs for pharmaceuticals. ${ }^{28,29}$

\subsection{Characterization of Polymers}

\subsubsection{Thermogravimetric Analysis}

Thermal stability of the synthesized polymers was studied using TGA. Based on results (Fig. 1), similar degradation patterns at different temperatures were observed. However, the MIP was observed to be more stable with its backbone collapsing at $355^{\circ} \mathrm{C}$, than the NIP which completely degraded at $255^{\circ} \mathrm{C}$. The differences in the degradation temperatures could be associated to the alterations that could have taken place during the treatment of polymers with organic solvents that resulted in template elution from the MIP. Initially below $90{ }^{\circ} \mathrm{C}$, there was a weight loss of $2 \%$ and $12 \%$, for MIP and NIP, respectively. This was attributed to the loss of methanol that could have been trapped in the polymers during the template removal process. ${ }^{30}$ Therefore, the thermal stability of these polymers were deemed to be acceptable since their applications are performed at room temperature.

\subsubsection{Solid-state Nuclear Magnetic Resonance}

The solid-state 13C CP/MAS NMR spectra for MIP and NIP depicted in Fig. 2 shows similar chemical shifts, which is an indication of identical structural arrangement due to similar synthetic conditions. The resonances in solid-state are depend-

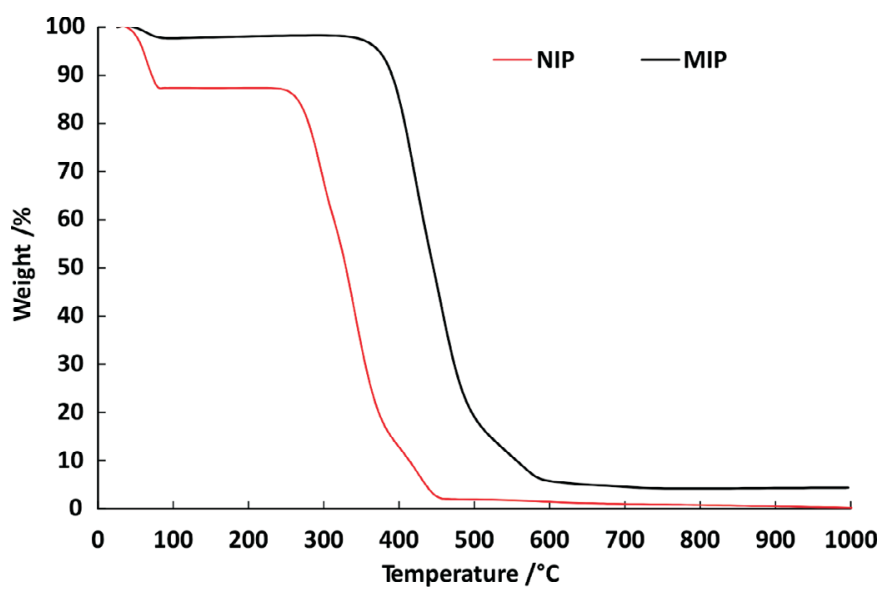

Figure 1 Thermogravimetric analysis of MIP and NIP.

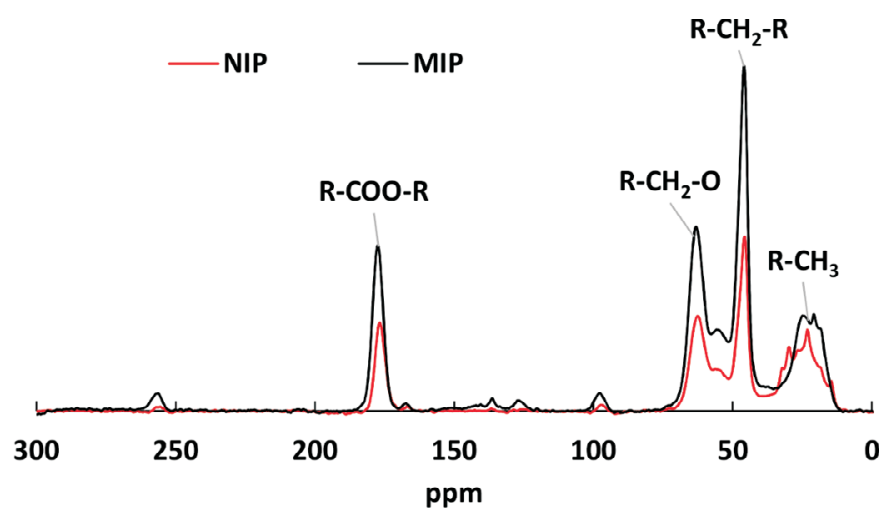

Figure 2 Solid-state 13C CP/MAS NMR Spectra of the MIP and NIP.

ent on the local arrangement of molecules and the signals of all polymer components can be detected. ${ }^{31}$ The strong signals for methyl (17 ppm), methylene (45 and $61 \mathrm{ppm}$ ) and $\mathrm{CO}_{2} \mathrm{R}$ (175 ppm) groups were observed. As reported in literature, ${ }^{12,32}$ these signals are normal for the polymers synthesized using large quantities of EGDMA as the cross-linking monomer. The weak resonances observed at $53 \mathrm{ppm}$ were due to aliphatic carbon atoms from EGDMA, while those at 124 and 136 ppm represented tertiary $\mathrm{CH}$ and quaternary carbon atoms, respectively. The spinning sidebands were observed at 100 and 255 ppm.

\subsubsection{Brunauer, Emmett and Teller Analysis}

The performance of the polymers during the adsorption of water pollutants is generally linked to their surface areas. Polymers of higher surface areas tend to be more effective in the adsorption of water pollutants. Both polymers synthesized in this study had a surface area of $372 \mathrm{~m}^{2} \mathrm{~g}^{-1}$ (Table 2) which is higher than the values reported previously by our research group. ${ }^{32,33}$ This means the alteration of the functional monomer without changing the synthetic approach or other reagents can modify the surface of the polymer. BET results (Table 2) further show that the removal of abacavir from the MIP did not alter its surface characteristics, hence the total pore volume and diameter are similar to those of the NIP. Average pore diameters for MIP and NIP were 4.33 and $4.44 \mathrm{~nm}$, respectively. These average pore diameters are in the range of 2 to $50 \mathrm{~nm}$, which means both polymers are mesoporous, which is common for the MIP and NIP that were synthesized for pharmaceuticals using the bulk polymerization approach. ${ }^{14,34}$

\subsection{Adsorption of Abacavir from Water: Optimization}

In this study, factors that have a potential to affect the adsorption of abacavir from polluted water using the synthesized MIP as the adsorbent were optimized. The investigated factors were the effect of sample $\mathrm{pH}$, contact time, MIP dosage amount and the initial concentration of abacavir in aqueous solutions. The initial concentration of abacavir in the aqueous solution was kept at $1 \mathrm{mg} \mathrm{L}^{-1}$ when the effects of sample $\mathrm{pH}$, contact time and MIP dosage amount were investigated. Although lower abacavir concentrations are expected in the aquatic environment, high

Table 2 Brunauer, Emmett and Teller analysis of polymers.

\begin{tabular}{lccc} 
Polymer & $\begin{array}{c}\text { Surface area } \\
/ \mathrm{m}^{2} \mathrm{~g}^{-1}\end{array}$ & $\begin{array}{c}\text { Total pore volume } \\
/ \mathrm{cm} \mathrm{g}^{-1}\end{array}$ & $\begin{array}{c}\text { Average pore diameter } \\
/ \mathrm{nm}\end{array}$ \\
\hline MIP & 372 & 0.403 & 4.33 \\
NIP & 372 & 0.413 & 4.44
\end{tabular}


levels of the drug were investigated for adsorption in order to determine the capacity of the adsorbent. In this way, the maximum amount of abacavir that can be adsorbed by $1 \mathrm{~g}$ of the adsorbent was determined. The adsorption was performed in the entire $\mathrm{pH}$ range (acidic, neutral and basic) in order to monitor the influence of structural variations that could occur in abacavir during the changes in sample $\mathrm{pH}$.

\subsubsection{Effect of Sample $p H$}

Optimization of sample $\mathrm{pH}$ is critical in the adsorption experiments as the $\mathrm{pH}$ tends to alter the chemical structure of the target molecule and the surface charge of the adsorbent. In this study, sample $\mathrm{pH}$ was varied over the range of $2-10$ while other experimental conditions were kept constant. Results portrayed in Fig. 3 clearly show that the sample $\mathrm{pH}$ plays a vital role in the adsorption of abacavir from water using the synthesized polymers. The extraction efficiencies for MIP exceeded $60 \%$ in the $\mathrm{pH}$ range of 5 to 10 , whereas, a sample $\mathrm{pH}$ of 2 was inefficient for the extraction of abacavir from aqueous solutions. This was expected as abacavir with pKa value of $5.06^{16,21}$ is likely to get protonated at the peripheral nitrogen $\left(\mathrm{NH}_{2}\right)$ when the $\mathrm{pH}$ is 2 and carry a positive charge $\left(\mathrm{NH}_{3}{ }^{+}\right)$. At the same time, aliquat 336 used as the functional monomer during the synthesis of both MIP and NIP has a permanent positive charge, which causes the electrostatic repulsion and result in poor extraction efficiency for abacavir at $\mathrm{pH}$ 2. When the sample $\mathrm{pH}$ is above 5 , abacavir is expected to exist as a neutral species that carries no charge. In this case $(\mathrm{pH}$ $\geq 5$ ), the electrostatic attraction between the peripheral nitrogen of abacavir and aliquat 336 is possible, hence high extraction efficiencies were achieved under these conditions. Furthermore, a similar electrostatic interaction could possibly occur due to the presence of oxygen atom in the abacavir molecule. This is expected throughout the $\mathrm{pH}$ range, which explains the low adsorption that occurred at $\mathrm{pH} 2$. In subsequent experiments, the $\mathrm{pH}$ of samples was adjusted to 5 , as this is the $\mathrm{pH}$ with maximum extraction efficiency for MIP.

\subsubsection{Effect of Contact Time}

The effect of contact time on adsorption of abacavir from aqueous solution was investigated by determining the extraction efficiency as a function of time. An extraction efficiency greater than $70 \%$ was achieved within $10 \mathrm{~min}$ of contact between the adsorbents and abacavir (Fig. 4). The extraction efficiency achieved for MIP increased gradually from $76 \%$ in the contact time of $10 \mathrm{~min}$ to $90 \%$ in $60 \mathrm{~min}$. There was no expressive improvement in the extraction efficiency of abacavir using

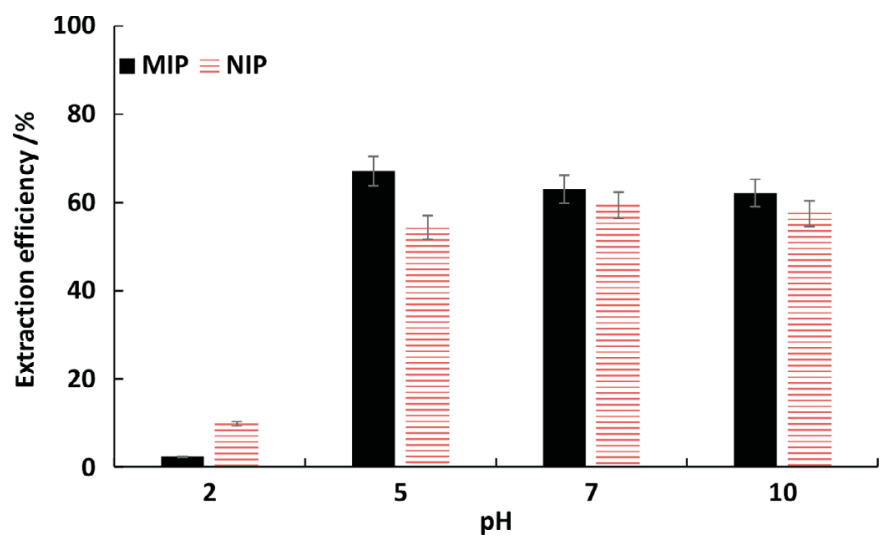

Figure 3 Effect of sample $\mathrm{pH}$ on extraction efficiency of abacavir Experimental details: polymer amount, abacavir concentration, sample volume and contact time were $20 \mathrm{mg}, 1 \mathrm{mg} \mathrm{L}^{-1}, 10 \mathrm{~mL}$ and $60 \mathrm{~min}$, respectively.

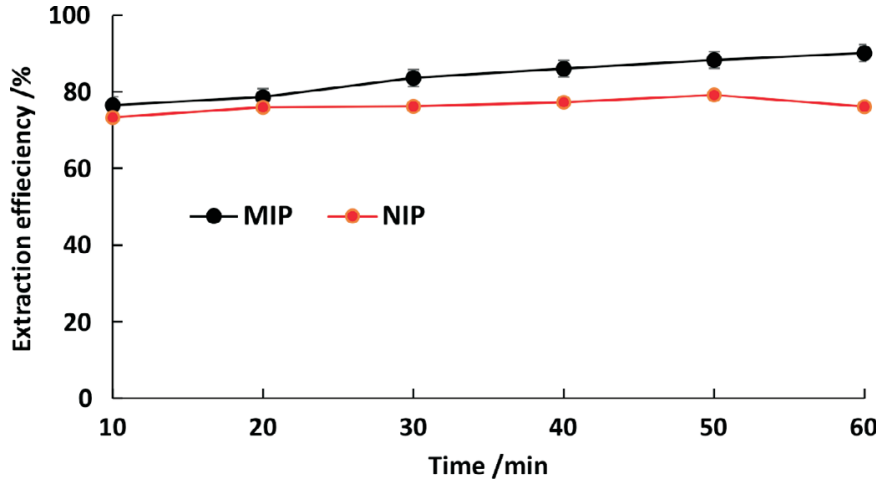

Figure 4 Effect of contact time on extraction efficiency of abacavir Experimental details: sample $\mathrm{pH}$, abacavir initial concentration, adsorbent mass and sample volume were $5,1 \mathrm{mg} \mathrm{L}^{-1}, 40 \mathrm{mg}$ and $10 \mathrm{~mL}$, respectively.

NIP after the contact time of $10 \mathrm{~min}$. This means the surface of the NIP reached its saturation point within $10 \mathrm{~min}$, while MIP had additional binding sites conformation on its surface suitable for abacavir adsorption due to the removal of template after synthesis.

\subsubsection{Effect of Dosage Amount}

The amount of polymer required for efficient adsorption of abacavir from water was investigated in the range of 10 to $50 \mathrm{mg}$. An increase in extraction efficiency for MIP from $60 \%$ to $92 \%$ was observed when the adsorbent mass was varied from 10 to $40 \mathrm{mg}$ (Fig. 5). There was no major variation in extraction efficiency when the adsorbent mass exceeded $40 \mathrm{mg}$, which resulted in selection of $40 \mathrm{mg}$ as the optimum weight. A similar trend was observed for the NIP. This means during the increase in adsorbent mass more binding sites in the polymer become available and the extraction efficiency is enhanced until the equilibrium was reached at a mass of $40 \mathrm{mg}$.

\subsubsection{Effect of Abacavir Initial Concentration}

The effect of abacavir initial concentration was investigated in the range of 10 to $50 \mathrm{mg} \mathrm{L}^{-1}$ and plotted against the adsorption capacity computed using Equation (2) as shown in Fig. 6. Results displayed in Fig. 6 show that the adsorption capacity increased linearly when the abacavir concentration was varied from $10 \mathrm{mg} \mathrm{L}^{-1}$ to $30 \mathrm{mg} \mathrm{L}^{-1}$. The equilibrium was reached at $30 \mathrm{mg} \mathrm{L}^{-1}$ with the corresponding adsorption capacities of $5.98 \mathrm{mg} \mathrm{g}^{-1}$ and $5.35 \mathrm{mg} \mathrm{g}^{-1}$ found using MIP and NIP as adsorbents, respectively. These results are in close agreement with the maximum adsorp-

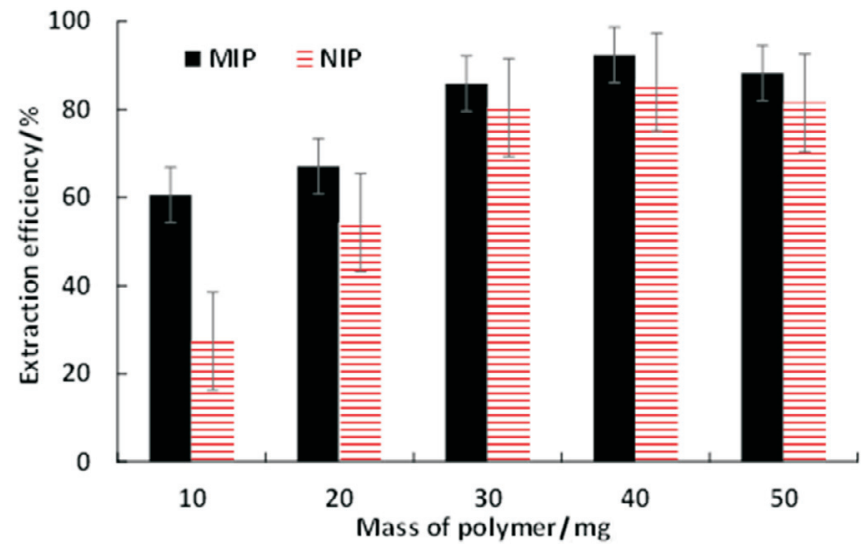

Figure 5 Effect of polymer mass on extraction efficiency of abacavir. Experimental details: sample $\mathrm{pH}$, abacavir concentration, sample volume and contact time were $5,1 \mathrm{mg} \mathrm{L}^{-1}, 10 \mathrm{~mL}$ and $60 \mathrm{~min}$, respectively. 




Figure 6 Effect of abacavir initial concentration on adsorption capacity. Experimental details: sample volume, $\mathrm{pH}$, adsorbent mass and contact time were $10 \mathrm{~mL}, 5,40 \mathrm{mg}$ and $60 \mathrm{~min}$, respectively.

tion capacity of $8.24 \mathrm{mg} \mathrm{g}^{-1}$ achieved for extraction of ketoprofen from water using a MIP prepared with similar reagents with the exception of the template molecule and 2-vinylpyridine that was used as the functional monomer in a previous study. ${ }^{32}$ Furthermore, this means $30 \mathrm{mg} \mathrm{L}^{-1}$ of abacavir could saturate $40 \mathrm{mg}$ of the investigated adsorbents.

However, this could not happen in the aquatic environment as reported in section 3.7 of this article, because low concentrations of abacavir are expected in the environmental waters. In recent work, the concentrations reported for abacavir in WWTPs located around the city of Durban in South Africa did not exceed $14 \mu \mathrm{g} \mathrm{L}-1.8$

\subsection{Binding Site Characterization}

\subsubsection{Kinetic Modelling}

In order to determine the adsorption mechanism, the kinetic data obtained in this study was processed using pseudo-firstorder and pseudo-second-order equations indicated as (3) and (4), respectively.

$$
\begin{aligned}
& \log \left(Q_{e}-Q_{t}\right)=\log Q_{e} \frac{k_{1} t}{2.303} \\
& \frac{\mathrm{t}}{\mathrm{Q}_{\mathrm{t}}}=\frac{1}{\mathrm{k}_{2} \mathrm{Q}_{\mathrm{e}}^{2}}+\frac{1}{\mathrm{Q}_{\mathrm{e}}} \mathrm{t}
\end{aligned}
$$

where $Q_{t}$ is the adsorption capacity $\left(\mathrm{mg} \mathrm{g}^{-1}\right)$ at time $t(\mathrm{~min}), Q_{e}$ is the adsorption capacity at equilibrium $\left(\mathrm{mg} \mathrm{g}^{-1}\right), \mathrm{k}_{1}$ and $\mathrm{k}_{2}$ are pseudo-first and second-order adsorption rate constants $\left(\mathrm{g} \mathrm{mg}^{-1} \mathrm{~min}^{-1}\right)$, respectively. The suitable adsorption model was determined based on the evaluation of the correlation coefficients $\left(R^{2}\right)$ of the polymers. The pseudo-second-order model was found to be the best fit (Table 3) yielding the adsorption capacities of 4.4 and $3.7 \mathrm{mg} \mathrm{g}^{-1}$ for MIP and NIP, respectively. These results imply that chemical adsorption onto the surface of MIP and NIP is responsible for the abatement of abacavir from aqueous solution. The equilibrium adsorption capacity reported for tetracycline using a MIP was $3.84 \mathrm{mg} \mathrm{g}^{-1} \cdot{ }^{35}$ Thus far, NIP gave lower extraction efficiencies for abacavir than MIP, which subse-

Table 3 Results of the kinetic modelling.

\begin{tabular}{lccccc}
\hline Polymer & \multicolumn{2}{c}{ Pseudo-first-order } & & \multicolumn{3}{c}{ Pseudo-second-order } \\
\cline { 2 - 3 } \cline { 5 - 6 } \cline { 5 - 6 } & $\mathrm{R}^{2}$ & & $\mathrm{R}^{2}$ & $\begin{array}{c}\mathrm{Q}_{\mathrm{e}} \\
/ \mathrm{mg} \mathrm{g}^{-1}\end{array}$ & $\begin{array}{c}\mathrm{k}_{2} \\
/ \mathrm{g} \mathrm{mg}^{-1} \mathrm{~min}^{-1}\end{array}$ \\
\hline MIP & 0.954 & 0.998 & 4.4 & 0.069 \\
NIP & 0.0473 & 0.996 & 3.7 & 0.37 \\
\hline
\end{tabular}

quently resulted in lesser adsorption capacity, therefore, it was omitted in other experiments.

\subsubsection{Adsorption Isotherms}

In order to acquire more insight on the nature of binding sites present in both MIP and NIP, isothermal analysis was investigated using Equations (5) and (6) which represent the linearized forms of Freundlich and Langmuir isotherms, respectively.

$$
\begin{aligned}
& \log \mathrm{Q}=\operatorname{mlog}_{\mathrm{e}}+\log \alpha \\
& \frac{\mathrm{C}_{\mathrm{e}}}{\mathrm{Q}}=\frac{\mathrm{C}_{\mathrm{e}}}{\mathrm{Q}_{\max }}+\frac{1}{\mathrm{Q}_{\max } \mathrm{K}_{\mathrm{L}}}
\end{aligned}
$$

where $\mathrm{Q}$ is the amount of the adsorbed molecule at equilibrium $\left(\mathrm{mg} \mathrm{g}^{-1}\right), \mathrm{m}$ is the adsorption intensity or surface heterogeneity, $\alpha$ is a Freundlich parameter related to the binding affinity, Ce is the equilibrium concentration of abacavir $\left(\mathrm{mg} \mathrm{L}^{-1}\right), \mathrm{Qmax}$ is the maximum adsorption capacity of abacavir $\left(\mathrm{mg} \mathrm{g}^{-1}\right)$ and $\mathrm{K}_{\mathrm{L}}$ is the Langmuir adsorption equilibrium constant.

The correlation coefficients $\left(\mathrm{R}^{2}\right)$ for Freundlich isotherm were 0.964 (MIP) and 0.974 (NIP), while those found for Langmuir isotherm were less than 0.300 . This means the data were best fitted with Freundlich isotherm, which implied a multilayer adsorption. Similar adsorption intensities of 0.91 and 0.93 for MIP and NIP, respectively, were observed. These values are close to 1 , which is an indication that the surfaces of both polymers are homogeneous. By description, adsorption intensities should vary between 0 and 1 , where 1 denotes to a totally homogeneous binding site distribution, with higher values corresponding to more homogeneous systems. ${ }^{36}$

\subsection{Selectivity Studies}

The selectivity of MIP for abacavir was investigated by adsorbing abacavir from a $10 \mathrm{~mL}$ water solution $(\mathrm{pH}$ 5) spiked with a mixture of abacavir, tenofovir disoproxil and efavirenz at $50 \mu \mathrm{g} \mathrm{L}^{-1}$ for each drug. All other optimum conditions (adsorbent mass of $40 \mathrm{mg}$ and contact time of $60 \mathrm{~min}$ ) for the adsorption process were utilized. The selected competitors (tenofovir disoproxil and efavirenz) are both ARVDs that contain functional groups such as amine groups and oxygen atoms, which are similar to those in the abacavir molecule (Table 1 ). The extraction efficiency for abacavir in the presence of these competitors was greater than $80 \%$, while tenofovir disoproxil and efavirenz had the extraction efficiencies of $60 \%$ and $29 \%$, respectively (Fig. 7). This means there was a strong competition between abacavir and tenofovir disoproxil for adsorption on a MIP. Tenofovir disoproxil has the pKa value of $4.13^{1}$, which means it would easily compete with abacavir (pKa 5.01) for adsorption on



Figure 7 Extraction efficiencies (MIP) of abacavir from polluted water containing tenofovir disoproxil and efavirenz $(n=3)$. 
MIP, while the $p K a$ value for efavirenz is -1.5 . Despite the strong interactions that could have occurred between the MIP and competitors, their lower extraction efficiencies are attributed to their molecular shapes and sizes (Table 1), which could prevent these molecules to firmly bind into the MIP adsorption sites. This is well explained in literature, where the adsorption of compounds into MIPs is reported to be strongly influenced by the functional groups present in the target molecule, molecular shape and size. ${ }^{15}$

\subsection{Reusability Studies}

After adsorption of abacavir, MIP was regenerated through washing with a mixture of methanol $(80 \%)$ and acetic acid (20\%), which removed abacavir from the binding sites. This was followed by proper rinsing with methanol. Thereafter, the regenerated MIP was applied in the adsorption of abacavir from aqueous solutions. As shown in Fig. 8, the extraction efficiency was greater than $90 \%$, after ten consecutive adsorption/desorption cycles. The reusability of the synthesized MIP for the adsorption of abacavir resemble a similar behaviour of other MIP reported in literature for extraction of pharmaceuticals, such as carbamazepine and clofibric acid. ${ }^{37}$ The reusability of MIP for metformin showed a $7 \%$ decline in its performance within five adsorption/desorption cycles. ${ }^{38}$ There was no variation in the extraction efficiency when a MIP for acetylsalicylic acid was reused six times. ${ }^{39}$ The ability to reuse the synthesized MIP ten times is an advantage over many other reported adsorbents (reviewed elsewhere) ${ }^{40}$ for removal of pharmaceuticals from water.



Figure 8 Extraction efficiencies obtained after each regeneration cycle of the MIP.

\subsection{Adsorption of Abacavir from Contaminated Water}

The detected concentrations of abacavir in wastewater influent, effluent and estuarine water were 41,24 and $22 \mu \mathrm{g} \mathrm{L}^{-1}$, respectively. These levels were adjusted by spiking the wastewater influent, effluent and estuarine water samples to reach a final concentration of $50 \mu \mathrm{g} \mathrm{L}^{-1}$ for abacavir. This was essential as MIPs are mostly applied as SPE sorbents where the analytes are extracted during the percolation of water samples into the cartridge. During the SPE sample loading, the concentration of analytes accumulate in the sorbent thereby resulting in pre-concentration. Therefore, in this study the concentration of abacavir in the collected samples was adjusted prior to adsorption to account for these conditions. The optimized adsorption conditions were applied to evaluate the uptake of abacavir from these aqueous solution using a MIP. The removal efficiencies achieved in wastewater influent, effluent and estuarine water were 67,74 and $76 \%$, respectively. The variations in these removal efficiencies across different samples could be influenced by the matrix effects. Wastewater influent had the lowest removal efficiency of $67 \%$ compared to $74 \%$ achieved for the effluent. This means the organic and inorganic materials that could be present in wastewater influent competed strongly with abacavir for adsorption on MIP. This could have resulted in the saturation of the binding sites. Some wastewater constituents are removed during the purification process, which results in water of better quality in the effluent. These results mean the MIP is able to adsorb abacavir from polluted water.

\section{Conclusion}

In this study, a MIP for selective extraction of abacavir from water was synthesized using abacavir, aliquat 336, EGDMA, toluene and 1,1-azobis-(cyclohexanecarbonitrile) as template molecule, functional monomer, cross-linking agent, porogen and initiator, respectively. The synthesized MIP selectively adsorbed abacavir from aqueous solution in the presence of tenofovir disoproxil and efavirenz. Abacavir was successfully removed from wastewater influent, effluent and estuarine water with extraction efficiencies reaching 67,74 and $76 \%$, respectively. Chemisorption through electrostatic interactions influenced the uptake of abacavir from water. The synthesized MIP can be reused up to 10 consecutive times without losing its extraction efficiency. This study demonstrated the ability of applying aliquat 336 as a functional monomer in the synthesis of a selective MIP that results in the uptake of abacavir from polluted water.

\section{Acknowledgements}

This work was supported by the National Research Foundation (NRF) of South Africa (Grant Numbers: 114415 and 118757). NRF and Department of Science and Technology are gratefully acknowledged for the Freestanding, Innovation and Scarce Skills Development Fund awarded for a student bursary (a Masters student) (Grant Number: 113769).

\section{References}

1 S. Ncube, L.M. Madikizela, L. Chimuka and M.M. Nindi, Environmental fate and ecotoxicological effects of antiretrovirals: a current global status and future perspectives, Water Res., 2018, 145, 231-247.

2 G.J. Yuen, S. Weller and G.E. Pakes, A review of the pharmacokinetics of abacavir, Clin. Pharmacokinet., 2008, 47, 351-371.

3 Y. Aminot, K. Le Menach, P. Pardon, H. Etcheber and H. Budzinski, Inputs and seasonal removal of pharmaceuticals in the estuarine Garonne River, Mar. Chem., 2016, 185, 3-11.

4 Y. Aminot, X. Litrico, M. Chambolle, C. Arnaud, P. Pardon and H. Budzindki, Development and application of a multi-residue method for the determination of 53 pharmaceuticals in water, sediment, and suspended solids using liquid chromatography-tandem mass spectrometry, Anal. Bioanal. Chem., 2015, 407, 8585-8604.

5 J. Funke, C. Prasse and T.A. Ternes, Identification of transformation products of antiviral drugs formed during biological wastewater treatment and their occurrence in the urban water cycle, Water Res., 2016, 98, 75-83.

6 C. Prasse, M.P. Schlüsener, R. Schulz and T.A. Ternes, Antiviral drugs in wastewater and surface waters: a new class of pharmaceuticals of environmental relevance?, Environ. Sci. Technol., 2010, 44, 1728-1735.

7 T.P. Wood, C.S.J. Duvenage and E. Rohwer, The occurrence of anti-retroviral compounds used for HIV treatment in South African surface water, Environ. Pollut., 2015, 199, 235-243.

8 O. Abafe, J. Spath, J. Fick, S. Jansson, C. Buckley, A. Stark, B. Pietruschka and B. Martincigh, LC-MS/MS determination of antiretroviral drugs in influents and effluents from wastewater treatment plants in KwaZulu-Natal, South Africa, Chemosphere, 2018, 200, 660-670. 
9 L.M. Madikizela, N.T. Tavengwa and L. Chimuka, Status of pharmaceuticals in African water bodies: occurrence, removal and analytical methods, J. Environ. Manage., 2017, 193, 211-220.

10 J. Akhtar, N.A.S. Amin and K. Shahzad, A review on removal of pharmaceuticals from water by adsorption, Desalin. Water Treat., 2016, 57, 12842-12860.

11 M. Cantarella, S.C. Carroccio, S. Dattilo, R. Avolio, R. Castaldo, C. Puglisi and V. Privitera, Molecularly imprinted polymer for selective adsorption of diclofenac from contaminated water, Chem. Eng. J., 2019, 367, 180-188.

12 L.M. Madikizela and L. Chimuka, Determination of ibuprofen, naproxen and diclofenac in aqueous samples using a multi-template molecularly imprinted polymer as selective adsorbent for solidphase extraction, J. Pharm. Biomed. Anal., 2016, 128, 210-215.

13 Z.E. Mbhele, S. Ncube and L.M. Madikizela, Synthesis of a molecularly imprinted polymer and its application in selective extraction of fenoprofen from wastewater, Environ. Sci. Pollut. Res., 2018, 25 36724-36735.

14 S.P. Mtolo, P.N. Mahlambi and L.M. Madikizela, Synthesis and application of a molecularly imprinted polymer in selective solid-phase extraction of efavirenz from water, Water Sci. Technol., 2019, 79, 356-365.

15 C. He, Y. Long, J. Pan, K. Li and F. Liu, Application of molecularly imprinted polymers to solid-phase extraction of analytes from real samples, J. Biochem. Biophys. Methods, 2007, 70, 133-150.

16 Z. Terzopoulou, M. Papageorgiou, G.Z. Kyzas, D.N. Bikiaris and D.A. Lambropoulou, Preparation of molecularly imprinted solid-phase microextraction fiber for the selective removal and extraction of the antiviral drug abacavir in environmental and biological matrices, Anal. Chim. Acta, 2016, 913, 63-75.

17 I. Chianella, K. Karim, E. V. Piletska, C. Preston and S.A. Piletsky, Computational design and synthesis of molecularly imprinted polymers with high binding capacity for pharmaceutical applications model case: adsorbent for abacavir, Anal. Chim. Acta, 2006, 559, 73-78.

18 A. Keshav, S. Chand and K.L. Wasewar, Recovery of propionic acid from aqueous phase by reactive extraction using quarternary amine (Aliquat 336) in various diluents, Chem. Eng. J., 2009, 152, 95-102.

19 H.V. Lerum, N.H. Andersen, D.Ø. Eriksen, E.W. Hansen, D. Petersen, G. Wibetoe and J.P. Omtvedt, Study of Cadmium extraction with Aliquat 336 from highly saline solutions, J. Solution Chem., 2018, 47, 1395-1417.

20 H. Yan, N. Sun, Y. Han, C. Yang, M. Wang and R. Wu, Ionic liquid-mediated molecularly imprinted solid-phase extraction coupled with gas chromatography-electron capture detector for rapid screening of dicofol in vegetables, J. Chromatogr. A., 2013, 1307, 21-26.

21 M.. Goizman, T.. Balayants, G.. Tikhomirova and N. Sal'nikova, Structure of chemical compounds. Methods of analysis and process control assay of abacavir sulfate, Pharm. Chem. J., 2012, 46, 187-191.

22 Y.P. Duan, C.M. Dai, Y.L. Zhang and Ling-Chen, Selective trace enrichment of acidic pharmaceuticals in real water and sediment samples based on solid-phase extraction using multi-templates molecularly imprinted polymers, Anal. Chim. Acta, 2013, 758, 93-100.

23 S. Matongo, G. Birungi, B. Moodley and P. Ndungu, Pharmaceutical residues in water and sediment of Msunduzi River, KwaZulu-Natal, South Africa, Chemosphere, 2015, 134, 133-140.

24 F.O. Agunbiade and B. Moodley, Pharmaceuticals as emerging organic contaminants in Umgeni River water system, KwaZulu-Natal, South Africa, Environ. Monit. Assess., 2014, 186, 7273-7291.

25 N.P. Ngubane, D. Naicker, S. Ncube, L. Chimuka and L.M Madikizela, Determination of naproxen, diclofenac and ibuprofen in Umgeni estuary and seawater: a case of northern Durban in
KwaZulu-Natal Province of South Africa, Reg. Stud. Mar. Sci., 2019, 29, 100675 .

26 L.M. Madikizela and L. Chimuka, Occurrence of naproxen, ibuprofen, and diclofenac residues in wastewater and river water of KwaZulu-Natal Province in South Africa, Environ. Monit. Assess., 2017, 189, 348.

27 C. Rimayi, D. Odusanya, J.M. Weiss, J. De Boer and L. Chimuka, Contaminants of emerging concern in the Hartbeespoort Dam catchment and the uMngeni River estuary 2016 pollution incident, South Africa, Sci. Total Environ., 2018, 627, 1008-1017.

28 L.M. Madikizela and L. Chimuka, Synthesis, adsorption and selectivity studies of a polymer imprinted with naproxen, ibuprofen and diclofenac, J. Environ. Chem. Eng., 2016, 4, 4029-4037.

29 L.M. Madikizela, N.T. Tavengwa and L. Chimuka, Applications of molecularly imprinted polymers for solid-phase extraction of non-steroidal anti-inflammatory drugs and analgesics from environmental waters and biological samples, J. Pharm. Biomed. Anal., 2018, $147,624-633$

30 S.S. Zunngu, L.M. Madikizela, L. Chimuka and P.S. Mdluli, Synthesis and application of a molecularly imprinted polymer in the solidphase extraction of ketoprofen from wastewater, Comptes Rendus Chim., 2017, 20, 585-591.

31 M. Sobiech, Ż. Teresa, P. Luli and D. Maciejewska, Separation of octopamine racemate on $(\mathrm{R}, \mathrm{S})$-2-amino-1-phenylethanol imprinted polymer - Experimental and computational studies, Talanta, 2016, 146, 556-567.

32 L.M. Madikizela, S.S. Zunngu, N.Y. Mlunguza, N.T. Tavengwa, P.S Mdluli and L. Chimuka, Application of molecularly imprinted polymer designed for the selective extraction of ketoprofen from wastewater, Water SA, 2018, 44, 406-418.

33 L.M. Madikizela, P.S. Mdluli and L. Chimuka, Experimental and theoretical study of molecular interactions between 2-vinyl pyridine and acidic pharmaceuticals used as multi-template molecules in molecularly imprinted polymer, React. Funct. Polym., 2016, 103,33-43.

34 K. Farrington and F. Regan, Investigation of the nature of MIP recognition: the development and characterisation of a MIP for Ibuprofen, Biosens. Bioelectron., 2007, 22, 1138-1146.

35 W. Cai and R.B. Gupta, Molecularly-imprinted polymers selective for tetracycline binding, Sep. Purif. Technol., 2004, 35, 215-221.

36 Y. Tang, J. Lan, X. Gao, X. Liu, D. Zhang, L. Wei, Z. Gao and J. Li, Determination of clenbuterol in pork and potable water samples by molecularly imprinted polymer through the use of covalent imprinting method, Food Chem., 2016, 190, 952-959.

37 C. Meng Dai, J. Zhang, Y. Lei Zhang, X. Fei Zhou, Y. Ping Duan and S. Guang Liu, Removal of carbamazepine and clofibric acid from water using double templates-molecularly imprinted polymers, Environ. Sci. Pollut. Res., 2013, 20, 5492-5501.

38 G.Z. Kyzas, S.G. Nanaki, A. Koltsakidou, M. Papageorgiou, M. Kechagia, D.N. Bikiaris and D.A. Lambropoulou, Effectively designed molecularly imprinted polymers for selective isolation of the antidiabetic drug metformin and its transformation product guanylurea from aqueous media, Anal. Chim. Acta, 2015, 866, $27-40$.

39 F. Meischl, D. Schemeth, M. Harder, N. Köpfle, R. Tessadri and M. Rainer, Synthesis and evaluation of a novel molecularly imprinted polymer for the selective isolation of acetylsalicylic acid from aqueous solutions, J. Environ. Chem. Eng., 2016, 4, 4083-4090.

40 N.Y. Mlunguza, S. Ncube, P.N. Mahlambi, L. Chimuka and L.M. Madikizela, Adsorbents and removal strategies of non-steroidal anti-inflammatory drugs from contaminated water bodies, J. Environ. Chem. Eng., 2019, 7, 103142. 